\title{
Efficacy and complications of different drainage tube fixing methods on percutaneous biliary drainage
}

\author{
Z.H. Peng, W.Y. Shi, Z. Xiong, B.S. Zhao, D.Z. Zhang, M.Q. Wang, \\ W. Song, K. Wang, B. Liu*
}

Department of Radiology, The First Affiliated Hospital of Anhui Medical University, Hefei, Anhui 230022, P.R. China

\begin{abstract}
Background: Percutaneous biliary drainage (PTBD) is a palliative treatment for malignant biliary obstruction. The present study adopted different methods of fixing the drainage tube, aiming to evaluate the efficacy and complications for patients. Materials and Methods: Total 68 patients with malignant biliary obstruction, enrolled from 2015 May to 2018 March, were randomly divided into two groups to perform PTBD. 34 patients in group A were fixed the drainage tube by using the traditional way and 34 patients in group B by using an ameliorated approach. The clinical success rate, level of direct/indirect bilirubin, complications and quality of life of patients were recorded. Results: PTBD was successfully performed on all 68 patients. The direct and indirect bilirubin levels of all patients were significantly decreased at 1 -week and 1-month post-surgery $(P<0.001)$. Compared with group $A$, the level of direct bilirubin in group $B$ decreased more significantly at 1-month after the operation $(P<0.05)$. The quality of life of patients was improved in the two groups at 1-month after the operation $(P<0.001)$. In addition, the rate of early postoperative complications was $38.24 \%$ in group $B$, which was not significantly different from the rate in group $A(41.18 \%)(P>0.05)$. However, the complication rate of late postoperative in group $B(6 / 34)$ was significantly less than complication rate in group A $(16 / 34)(P<0.05)$. Conclusion: PTBD is an effective method of treating malignant biliary stricture. The ameliorated fixing method for the drainage tube in PTBD effectively decreases the incidence of late complications, further improving patient quality of life.
\end{abstract}

Keywords: percutaneous biliary drainage, malignant biliary obstruction, complications

\section{INTRODUCTION}

At present, the technique of percutaneous biliary drainage (PTBD) is recognized as an effective treatment for malignant biliary obstruction $(1,2)$, which was first reported in 1974 (3) and has been widely used in clinical practice for over 40 years (4). This method can improve quality of life for patients to varying degrees, but some complications cannot be avoided, even when the procedure is performed by an experienced interventional radiologist (5). These complications may include abdominal pain, hemorrhage, biliary peritonitis, cholangitis, pancreatitis and electrolyte disturbances $(5,6)$.
Complications occurring shortly after the procedure can be dealt with immediately, as the patient will still be in hospital. There have been a lot of previous reports with regard to how to treat complications appearing during the early postoperative periods. However, only a few studies exist about how to prevent complications caused by the drainage tube becoming dislocated/falling off, or delayed puncture point infections after the patient is discharged from hospital. In order to ensure the drainage effect, the biliary drainage tube needs to be properly fixed and managed (7). The traditional fixation device does not fix the wound with sutures, and the patient need to 
carry drainage bag when gets out of bed (8). If scratching or drainage penetration occurs, the catheter can be pulled which lead to reduced patient activity and increases infection Risk (6).

The present study retrospectively analyzed 68 patients with malignant biliary obstruction from May 2015 to March 2018 in First Affiliated Hospital of Anhui Medical University. New methods of biliary drainage tube fixation kit were introduced, which can significantly reduce the risk of catheter pulling and dislocation caused by poor drainage tube fixation during daily activities, and reduce the risk of skin dislocation in preliminary investigation. All patients underwent PTBD, and different methods of the drainage tube fixing were adopted. The occurrence of late complications and the improvement on quality of life were investigated.

\section{MATERIALS AND METHODS}

\section{Patients}

A total of 68 patients with malignant biliary obstruction enrolled from May 2015 to March 2018 in the First Affiliated Hospital of Anhui Medical University were randomly divided into two groups. All patients were treated with PTBD by an experienced interventional radiologist. In group A, 34 patients were treated using the traditional way of fixing the drainage tube. In group B, 34 patients had their drainage tubes fixed with an ameliorated approach. The baseline characteristics of the patients are summarized in table 1. All experiments involving clinical patient samples were performed in accordance with a protocol approved by the Ethics Committee of The First Affiliated Hospital of Anhui Medical University, China. Written informed consent was obtained from each patient before the operation. All methods were performed in accordance with the relevant guidelines and regulations.

\section{Procedure}

Before PTBD, all patients were examined by imaging, electrocardiography and laboratory testing. The imaging examination included contrast-enhanced thoracoabdominal computed tomography or/and magnetic resonance (German, Siemens) cholangiopancreatography (MRCP) in order to ascertain the degree of dilation and obstructive site of the bile duct, and the present/absence of ascites. The biochemical analyses, complete blood count and prothrombin time were measured before the procedure to assess indications and contraindications. The Karnofsky performance status (KPS) questionnaire was implemented in the two groups for the evaluation of preoperative quality of life.

The basic operational approach for PTBD was first reported by published methods ${ }^{(9)}$. In the present study, digital subtraction angiography (DSA)-guided percutaneous transhepatic puncture cholangiography was first performed using a 22-gauge needle to clear the bile duct branch (figure 1). Subsequently, the best puncture pathway was selected in order to obtain the optimal location for the drainage tube. After successful puncture, an 8F bile duct drainage tube was inserted in the bile duct under the guidance of a guidewire; when bile was aspirated through the drainage tube, the procedure was regarded as successful. In group $A$, the traditional method was used to fix drainage tube (figure 2): the end of the drainage tube was connected to the drainage bag, suture binding was performed, and then a bandage dressing was directly applied. In group B, the drainage tube was fixed with an ameliorated approach (figure 3): After suture binding of the drainage tube, it was inserted into a stoma bag, which was then fixed in place. At length, we used the drainage bag connected with the stoma bag. Post-operation complications were divided into early and late. The early complications included bleeding, abdominal pain, nausea, vomiting, cholangitis and peritonitis. The late complications included drainage tube dislocation and delayed puncture point infections (figure 4).

\section{Follow up}

Laboratory examination was performed on all the patients at 1-week and 1-month after surgery to investigate the level of direct bilirubin

Int. J. Radiat. Res., Vol. 19 No. 4, October 2021 
and indirect bilirubin. The incidences of early and late complications were observed postoperatively in both groups. Early complications usually occurred during hospitalization, and late complications were identified by clinical follow up within 1-month post-discharge. For patients with recurrent jaundice, drainage tube cholangiography was carried out to ascertain the location of drainage tube. The quality of life of all patients after PTBD was evaluated through KPS score, also within 1-month, as PTBD remains an important factor that enhances the quality of life of tumor patients.

\section{Statistical analysis}

Clinical data analysis was performed using SPSS version 19.0 software (IBM Corp). The Wilcoxon test was used to calculate the bilirubin levels and KPS scores between group A and group B. The $\chi^{2}$ test or fisher exact test was performed for the evaluation of early and late complications between the two groups. Univariate analysis was applied in each group separately to investigate the treatment effects. All statistical tests were two tailed, and $\mathrm{P}<0.05$ was considered to a statistically significant difference.

Table 1. Clinical characteristics of 68 patients.

\begin{tabular}{|c|c|c|c|}
\hline \multirow{2}{*}{ characteristics } & \multicolumn{2}{|c|}{ patients } & \\
\cline { 2 - 4 } & Group A & Group B & P value \\
\hline Gender (Male/Female) & $22 / 12$ & $16 / 18$ & 0.222 \\
\hline Age & $71.5(48 \sim 86)$ & $69.5(44 \sim 87)$ & 0.314 \\
\hline Primary lesion & & & \\
\hline pancreatic cancer & 3 & 5 & 0.709 \\
\hline Ampullar cancer & 4 & 2 & 0.673 \\
\hline Bile duct carcinoma & 9 & 11 & 0.79 \\
\hline Hepatocelluar carcinoma & 4 & 3 & 0.99 \\
\hline Metastatic tumor & & & \\
\hline Gastric cancer & 9 & 7 & 0.775 \\
\hline Colon cancer & 5 & 6 & 0.99 \\
\hline Drainage type & & & 0.476 \\
\hline Right/Left & $28 / 6$ & $31 / 3$ & \\
\hline Site of bile duct obstruction & & & 0.80 \\
\hline Proximal/Distal & $11 / 23$ & $13 / 21$ & 0.99 \\
\hline Concomitant of ascites & 4 & 3 & \\
\hline
\end{tabular}
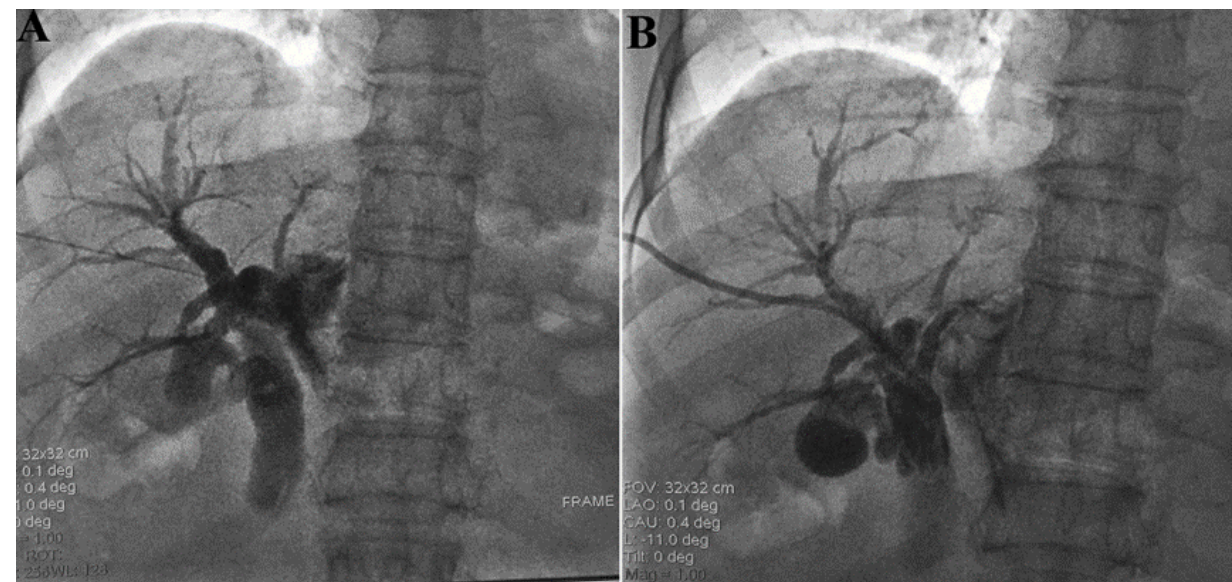

Figure 1. PTBD procedure. The PTBD needle was inserted into the intrahepatic bile duct. Contrast medium was injected into the bile duct to clearly show the biliary tree, and then the drainage tube was placed into the common bile duct with the guidance of a guidewire. PTBD, percutaneous biliary drainage. 


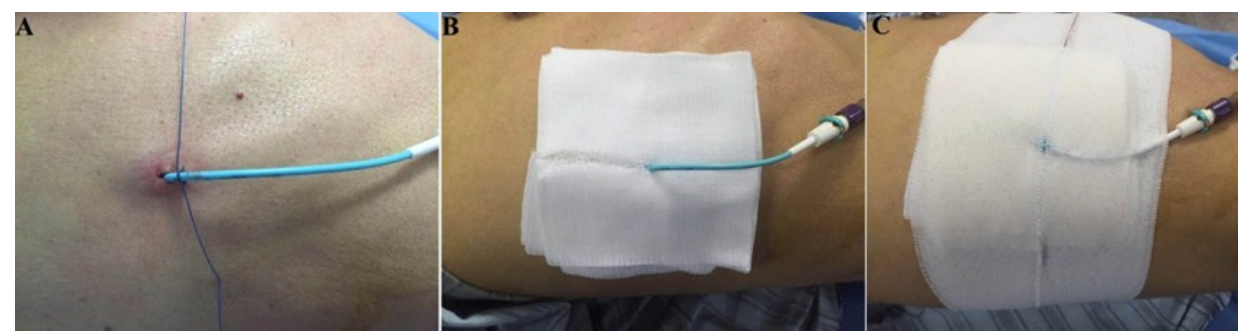

Figure 2. Traditional fixing method. After the drainage tube was sutured and fixed (A), a gauze (B) and adhesive tape dressing (C) was applied to the drainage tube, which was then directly connected to the drainage bag.

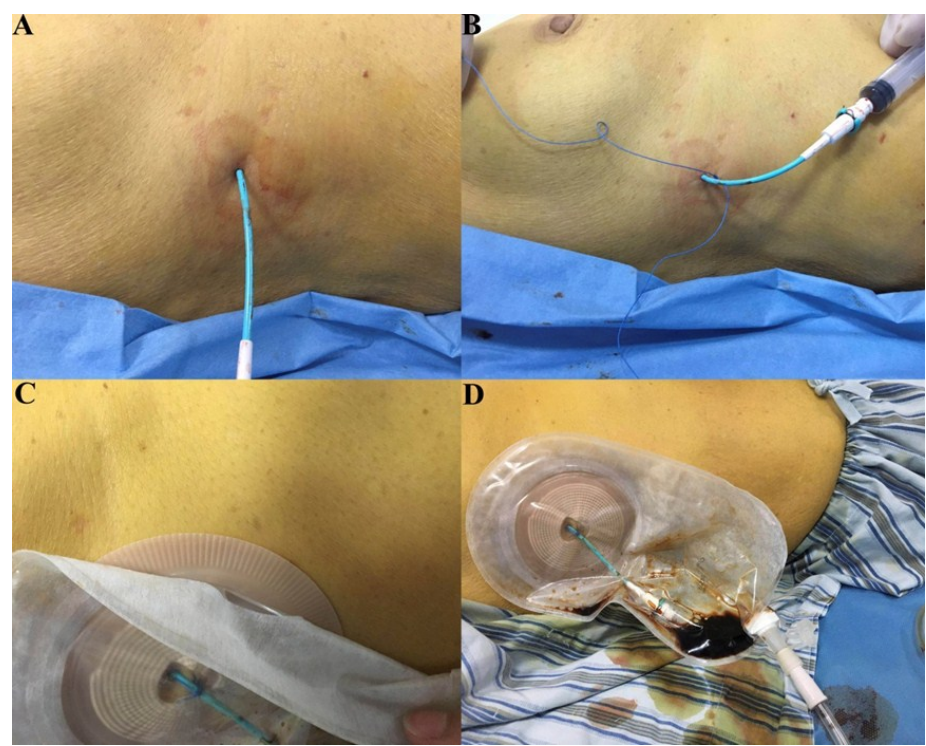

Figure 3. Ameliorated fixing method. The drainage tube was inserted into the stoma bag (A) after the tube had been dressed and fixed (B). The stoma bag was attached (C), and then used to connect to the drainage bag (D).

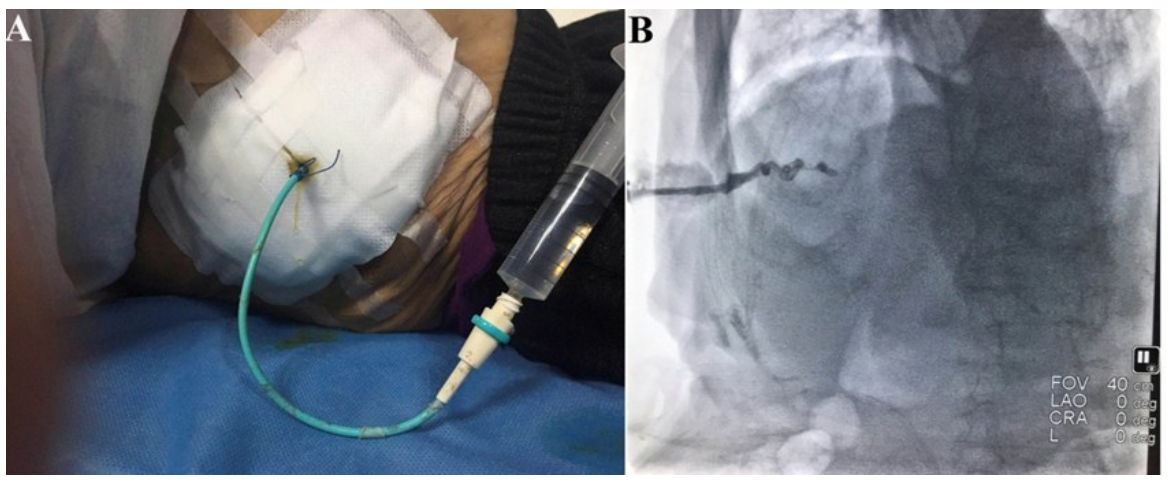

Figure 4. Complications. In this patient, the drainage tube was attached using the traditional fixing method. Drainage tube dislocation occurred at 1 month after percutaneous biliary drainage.

\section{RESULTS}

Clinical characteristics including gender distribution, age, primary lesion, metastatic tumor, drainage, obstruction site and concomitant of ascites show no significant difference between two groups (table 1).

PTBD was successfully performed on all 68 950 patients. A total of 59 patients underwent right, and 9 patients underwent left drainage. The drainage tube fixing in half of patients were used traditional methods (figure 2) and half of them used ameliorated methods (figure 3). The direct and indirect bilirubin levels of all patients were significantly decreased after 1 -week and 1 -month (figure $5 \mathrm{~A}$ and $5 \mathrm{~B}, \mathrm{P}<0.001$ ). Compared 
with group A, the level of direct bilirubin in group $B$ decreased more significantly at 1 -month after surgery (figure $5 \mathrm{~A}, \mathrm{P}<0.05$ ). The level of indirect bilirubin was shown no significant difference between two groups either 1-week or 1-month later (figure 5B). In group A, drainage tube dislocation occurred in 5 patients, and the tube had fallen off in 6 patient's 1-month after surgery and these factors may lead to poor drainage.

The quality of life of patients was improved in the two groups at 1-month after the operation (figure $5 \mathrm{C}, \mathrm{P}<0.001$ ). The result for group $\mathrm{B}$ was superior to that for group $\mathrm{A}$, because a minority of patients experienced recurrent bilirubin in group A owing to poor drainage (figure 5C, $\mathrm{P}<0.001)$. In addition, the traditional drainage tube fixation method also affects the quality of life of patients. The rate of early postoperative complications was 38.2\% (13/34) in Group B, which was not significantly different from that in group A $(41.2 \%, 14 / 34)$ (figure 5D). However, the rate of late postoperative complications was $17.6 \%(6 / 34)$ in Group B, which was significantly less than that in group $A$ $(47.1 \%, 16 / 34)$ (figure 5D, $\mathrm{P}<0.05)$.

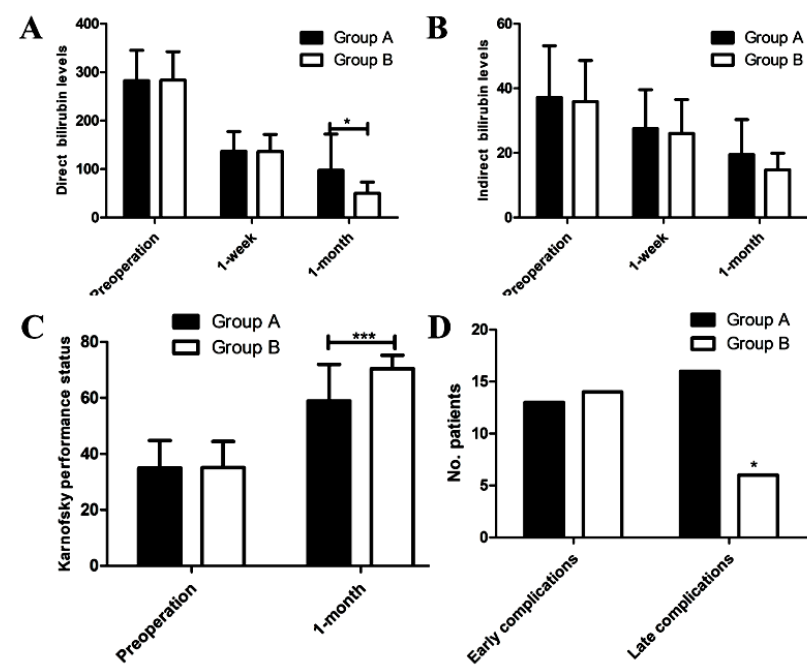

Figure 5. Comparison of direct (A) and indirect bilirubin (B) levels among preoperation, 1-week, and 1-month after surgery in two groups. Comparison of KPS (C) between preoperation and 1-month after surgery in two groups. Comparison number of patients (D) between early and late complications in two groups.

Int. J. Radiat. Res., Vol. 19 No. 4, October 2021

\section{DISCUSSION}

Malignant obstructive jaundice usually results from direct invasion or external compression caused by malignant tumors, including pancreatic cancer, ampullary cancer, bile duct carcinoma, hepatocellular carcinoma and metastatic tumors. Few patients are eligible for surgery (10). In order to enhance quality of life and prolong life, patients usually have no choice but to accept palliative biliary drainage. The current study indicated that the quality of life of patients was enhanced in groups with ameliorated fixing method.

Undoubtedly, PTBD plays a significant role in the treatment of biliary malignancies. However, PTBD-related complications are often inevitable. Therefore, the control of these complications is of importance. According to the Interventional Radiology Association, these complications may be divided into major and minor complications (11). In the present study, the complications were divided into early and late complications based on the time frame in which they occurred. It is reported that $21-77 \%$ of patients will experience complications as a result of a PTBD procedure (5, 12, 13). Cholangitis, abdominal pain and dislocation of the drainage tube are the most common complications after biliary drainage. Reasons for infection usually including low immunity in patients, or retrograde entry of intestinal bacteria into the biliary duct, which can have serious consequences (14-16). In the present study, 4 patients developed an infection in group $\mathrm{A}$, and 3 patients developed an infection in group B after PTBD. Fortunately, no serious complications occurred, due to timely anti-infective treatment. Generally, infection occurred within 1-week after surgery, and thus was defined as an early complication; as the patients were still in hospital, treatment was readily available.

Drainage tube dislocation includes the tube falling off or prolongating, leading to drainage failure or incomplete drainage $(17,18)$ and causing obstructive jaundice to recur in the patient. In current study, the traditional method of fixing the drainage tube was used in group $\mathrm{A}$, and an ameliorated approach in group B. After 1-month 
follow up, it was observed that the incidence of tube dislocation in group A was higher than that in group B. In the traditional method of fixing the drainage tube, the drainage bag connection tube may directly pull on the drainage tube. If the patient does not pay attention to protecting the drainage tube, once pulled by an external force it will prolongate outward or fall off. However, in group B, an improved drainage tube fixation and drainage method was applied. The first step involved insertion of the drainage tube and suspension within a stoma bag. The stoma bag was attached to the skin, and then the drainage bag connection tube was connected to the stoma bag; therefore, the drainage bag cannot directly pull on the drainage tube. According to the present observations, one patient experienced drainage tube dislocation in group $\mathrm{B}$, but the incidence of drainage tube dislocation was comparatively high in group A. Patients usually require a repeated drainage procedure if the drainage tube falls off, although a small number of patients in the past have had stents placed at the site of obstruction (19-21).

As a palliative therapeutic method, PTBD also improves patient quality of life and survival time (10). According to the follow-up data, the patient quality of life was improved to a certain extent in both groups in the present study, compared with the preoperative scores. However, the presence of an external drainage tube will have some negative impacts on daily life, such as the inability to bathe and the effect on sleeping posture. For patients with concurrent ascites, the ascites may exude along the sinus, saturating the gauze and clothes. On one hand, this will lead to decrease in patient quality of life, on the other hand it may lead to local infection. For patients with ascites, the exudates from the ascites will flow directly into the stoma bag directly. Since the stoma bag is securely attached and is water proof, patients may bathe, and move freely whilst asleep.

\section{CONCLUSION}

In conclusion, PTBD is an effective method of alleviating malignant biliary stricture. An ameliorated drainage tube fixation method can improve patient quality of life and reduced the incidence of complications compared with traditional fixing methods.

\section{ACKNOWLEDGMENTS}

The study was supported by the Department of Radiology of the First Affiliated Hospital of Anhui Medical University. The authors would like to express thanks to the technicians, nurses and other staff of the department for their assistance.

\section{Conflicts of interest: Declared none.}

\section{REFERENCES}

1. Schoenemann J, Willems M, Wolf G, Fromme M (1987) Results of percutaneous transhepatic drainage of the bile ducts. RoFo: Fortschritte auf dem Gebiete der Rontgenstrahlen und der Nuklearmedizin, 147(6): 619-23.

2. Teixeira MC, Mak MP, Marques DF, Capareli F, Carnevale FC, Moreira AM, et al. (2013) Percutaneous transhepatic biliary drainage in patients with advanced solid malignancies: prognostic factors and clinical outcomes. Journal of gastrointestinal cancer, 44(4): 398-403.

3. Molnar W and Stockum AE (1974) Relief of obstructive jaundice through percutaneous transhepatic catheter-a new therapeutic method. American Journal of Roentgenology, 122(2): 356-67.

4. Sharaiha RZ, Khan MA, Kamal F, Tyberg A, Tombazzi CR, Ali $B$, et al. (2017) Efficacy and safety of EUS-guided biliary drainage in comparison with percutaneous biliary drainage when ERCP fails: a systematic review and metaanalysis. Gastrointestinal endoscopy, 85(5): 904-14.

5. Duan F, Cui L, Bai Y, Li X, Yan J, Liu X (2017) Comparison of efficacy and complications of endoscopic and percutaneous biliary drainage in malignant obstructive jaundice: a systematic review and meta-analysis. Cancer Imaging, 17 (1): 1-7.

6. Lynn PB, Warnack EM, Parikh M, Welcome AU (2020) Hemobilia as a Complication of Transhepatic Percutaneous Biliary Drainage: a Rare Indication for Laparoscopic Common Bile Duct Exploration. Journal of Gastrointestinal Surgery, 2020 Jun 30. doi: 10.1007/s11605-020-04664-w.

7. Gurusamy KS, Koti R, Davidson BR (2013) T-tube drainage versus primary closure after open common bile duct exploration. Cochrane Database Syst Rev, (6): CD005640.

8. Zheng SM, Li H, Li GC, Yu DS, Ying DJ, Zhang B, et al. (2017) Risk factors, treatment and impact on outcomes of bile

Int. J. Radiat. Res., Vol. 19 No. 4, October 2021 
leakage after hemihepatectomy. ANZ Journal of Surgery, 87(7-8): E26-E31.

9. Fang $Y$, Gurusamy KS, Wang $Q$, Davidson BR, Lin $H$, Xie $X$, et al. (2012) Pre-operative biliary drainage for obstructive jaundice. Cochrane Database of Systematic Reviews, 9(9): CD005444.

10. Skipworth J, Olde Damink S, Imber C, Bridgewater J, Pereira S, Malagó M (2011) Surgical, neo-adjuvant and adjuvant management strategies in biliary tract cancer. Alimentary pharmacology \& therapeutics, 34(9): 1063-78.

11. Saad WE, Wallace MJ, Wojak JC, Kundu S, Cardella JF (2010) Quality improvement guidelines for percutaneous transhepatic cholangiography, biliary drainage, and percutaneous cholecystostomy. Journal of Vascular and Interventional Radiology, 21(6):789-95.

12. Nennstiel S, Weber A, Frick G, Haller B, Meining A, Schmid $\mathrm{RM}$, et al. (2015) Drainage-related complications in percutaneous transhepatic biliary drainage. Journal of CLinical Gastroenterology, 49(9): 764-70.

13. Ma J, Luo J, Gu J, Liu Q, Liu L, Zhang W, et al. (2018) Malignant obstructive jaundice treated with intraluminal placement of lodine-125 seed strands and metal stents: An analysis of long-term outcomes and prognostic features. Brachytherapy, 17(4): 689-95.

14. Dambrauskas Ž, Paškauskas $S$, Lizdenis $P$, Uktveris R, Pranculis A, Kaupas RS, et al. (2008) Percutaneous transhepatic biliary stenting: the first experience and results of the Hospital of Kaunas University of Medicine. Medicina, 44 (12): 969

15. Namias N, Demoya M, Sleeman D, Reever CM, Raskin JB, Ginzburg E, et al. (2005) Risk of postoperative infection in patients with bactibilia undergoing surgery for obstructive jaundice. Surgical Infections, 6(3): 323-8.

16. Herzog $T$, Belyaev $O$, Hessam $S$, Suelberg $D$, Janot $M$, Schrader H, et al. (2012) Bacteribilia with resistant microorganisms after preoperative biliary drainage-the influence of bacteria on postoperative outcome. Scandinavian Journal of Gastroenterology, 47(7): 827-35.

17. van Delden OM and Laméris JS (2008) Percutaneous drainage and stenting for palliation of malignant bile duct obstruction. European Radiology, 18(3): 448.

18. Nimura Y, Kamiya J, Kondo S, Nagino M, Uesaka K, Oda K, et al. (2000) Aggressive preoperative management and extended surgery for hilar cholangiocarcinoma: Nagoya experience. Journal of Hepato-Biliary-Pancreatic Surgery, 7(2): 155-62.

19. Brountzos EN, Ptochis N, Panagiotou I, Malagari K, Tzavara C, Kelekis D (2007) A survival analysis of patients with malignant biliary strictures treated by percutaneous metallic stenting. Cardiovascular and Interventional Radiology, 30(1): 66-73.

20. Pinol V, Castells A, Bordas JM, Real MI, Llach J, Montañà X, et al. (2002) Percutaneous self-expanding metal stents versus endoscopic polyethylene endoprostheses for treating malignant biliary obstruction: randomized clinical trial. Radiology, 225(1): 27-34.

21. Tsuyuguchi T, Takada T, Miyazaki M, Miyakawa S, Tsukada $K$, Nagino M, et al. (2008) Stenting and interventional radiology for obstructive jaundice in patients with unresectable biliary tract carcinomas. Journal of Hepato-BiliaryPancreatic Surgery, 15(1): 69. 
